02

\title{
Термическая стабильность поверхностного плазмонного резонансного поглощения в нанокомпозитных пленках $a-\mathrm{C}: \mathrm{H}\left\langle\mathrm{Ag}+\mathrm{TiO}_{2}\right\rangle$
}

\author{
() О.Ю. Приходько, С.Л. Михайлова , Е.С. Мухаметкаримов, К. Даутхан, \\ С.Я. Максимова, Г.А. Исмайлова, К.А. Тауасаров, Л.В. Михайлов
}

Казахский Национальный университет им. аль-Фараби, ННЛОТ, 050040 Алматы, Казахстан

T e-mail: Svetlana.Mikhailova@kaznu.kz

Поступила в редакцию 13.08.2018г.

Исследована термостабильность поверхностного плазмонного резонанса в нанокомпозитных пленках $a-\mathrm{C}: \mathrm{H}\langle\mathrm{Ag}\rangle$ и $a-\mathrm{C}: \mathrm{H}\left\langle\mathrm{Ag}+\mathrm{TiO}_{2}\right\rangle$. Пленки осаждали методом ионно-плазменного магнетронного распыления комбинированной графито-металлической мишени в атмосфере смеси аргона и метана. Структура и оптические свойства нанокомпозитных пленок изучались с помощью просвечивающей электронной микроскопии и оптической спектроскопии соответственно. Было обнаружено, что поверхностный плазмонный резонанс в пленках $a$-C: $\mathrm{H}\left\langle\mathrm{Ag}+\mathrm{TiO}_{2}\right\rangle$ сохраняется до температуры отжига $450^{\circ} \mathrm{C}$ в атмосфере аргона, тогда как в пленках $a$-C: $\mathrm{H}\langle\mathrm{Ag}\rangle$ поверхностный плазмонный резонанс исчезает при температуре отжига $350^{\circ} \mathrm{C}$. Было показано, что отжиг при $350^{\circ} \mathrm{C}$ приводит к значительному увеличению диаметра наночастиц серебра в структуре пленок $a-\mathrm{C}: \mathrm{H}\langle\mathrm{Ag}\rangle$. В пленках $a-\mathrm{C}: \mathrm{H}\left\langle\mathrm{Ag}+\mathrm{TiO}_{2}\right\rangle$ наряду с появлением наночастиц серебра с большим диаметром присутствуют наночастицы серебра с малым диаметром, которые сохраняются после отжига при этой температуре. Более высокая термическая стабильность поверхностного плазмонного резонанса в пленках $a$-C: $\mathrm{H}\left\langle\mathrm{Ag}+\mathrm{TiO}_{2}\right\rangle$ объясняется наличием наночастиц $\mathrm{TiO}_{2}$ в структуре пленок, которые ингибируют коалесценцию наночастиц серебра при высоких температурах.

DOI: $10.21883 /$ OS.2018.12.46935.238-18

Пленочные нанокомпозиты, состоящие из диэлектрической матрицы с внедренными наночастицами серебра, вызывают особый интерес, связанный с наличием у таких структур поверхностного плазмонного резонанса (ППР) в видимой области спектра [1,2]. Оптические свойства таких пленок перспективны для применения в оптических переключателях [3], ограничителях [4] и поглотителях $[5,6]$ для телекоммуникационных систем.

В качестве диэлектрической матрицы для наночастиц серебра используют $\mathrm{TiO}_{2}$ [7-10], $\mathrm{SiO}_{2}[11], \mathrm{Al}_{2} \mathrm{O}_{3}[12,13]$, $\mathrm{ZrO}_{2}$ [14-17], DLC (алмазоподобный углерод) [18] и $a-\mathrm{C}: \mathrm{H}[2]$. Эти матрицы различаются оптической шириной запрещенной зоны, диэлектрической проницаемостью и механическими свойствами. Положение максимума и полуширина пика ППР зависят от среднего диаметра наночастиц, их объемной доли в матрице и диэлектрической проницаемости матрицы [19]. Для практического использования пленочных нанокомпозитов с наночастицами серебра важным фактором является термическая стабильность ППР [20-23]. Так, например, в работе [18] показано, что отжиг пленок Ag:DLC при температуре $400^{\circ} \mathrm{C}$ приводит к уменьшению полуширины пика резонансного поглощения, голубому сдвигу ППР и появлению квадрупольного ППР. Наблюдаемые изменения связывают с коагуляцией наночастиц серебра и графитизацией матрицы DLC. После отжига композитов $\mathrm{Ag}: \mathrm{TiO}_{2}$ при $450^{\circ} \mathrm{C}$ ППР почти полностью исчезает [23].
Ранее [24] мы обнаружили, что пленки $a-\mathrm{C}: \mathrm{H}$, одновременно модифицированные $\mathrm{Ag}$ и Тi методом ионно-плазменного магнетронного распыления, имеют гетерофазную структуру, состоящую из матрицы $a-\mathrm{C}: \mathrm{H}$, изолированных наночастиц неокисленного серебра и аморфного диоксида титана со средним диаметром 2-3 nm. Кроме того, в нанокомпозитных пленках $a$-C: $\mathrm{H}\left\langle\mathrm{Ag}+\mathrm{TiO}_{2}\right\rangle$ наблюдались наночастицы серебра диаметром 30-60 nm. Нанокомпозитные пленки $a-\mathrm{C}: \mathrm{H}\left\langle\mathrm{Ag}+\mathrm{TiO}_{2}\right\rangle$, так же как и $a-\mathrm{C}: \mathrm{H}\langle\mathrm{Ag}\rangle$, обладали ярко выраженным ППР в видимой области спектра [24,25]. В настоящей работе представлены результаты сравнительного изучения термической стабильности ППР в нанокомпозитных пленках $a-\mathrm{C}: \mathrm{H}\langle\mathrm{Ag}\rangle$ и $a-\mathrm{C}: \mathrm{H}\left\langle\mathrm{Ag}+\mathrm{TiO}_{2}\right\rangle$.

\section{Методика эксперимента}

Пленочные нанокомпозиты $a-\mathrm{C}: \mathrm{H}\langle\mathrm{Ag}\rangle$ и $a-\mathrm{C}: \mathrm{H}\langle\mathrm{Ag}+$ $\left.+\mathrm{TiO}_{2}\right\rangle$ получали методом ионно-плазменного магнетронного распыления комбинированной мишени из пиролитического графита, серебра и титана в атмосфере смеси газов аргона и метана. Пленки нанокомпозитов осаждались на кремниевые (100) и кварцевые (KU-1) подложки со скоростью $2.7-3.3 \mathrm{~nm} / \mathrm{min}$. Температура осаждения составляла $100^{\circ} \mathrm{C}$ при плотности мощности разряда $0.6 \mathrm{~W} / \mathrm{cm}^{2}$. Толщина пленок варьировалась от 80 до $100 \mathrm{~nm}$ и определялась сканированием скола структуры $c$-Si/нанокомпозит на сканирующем электронном 

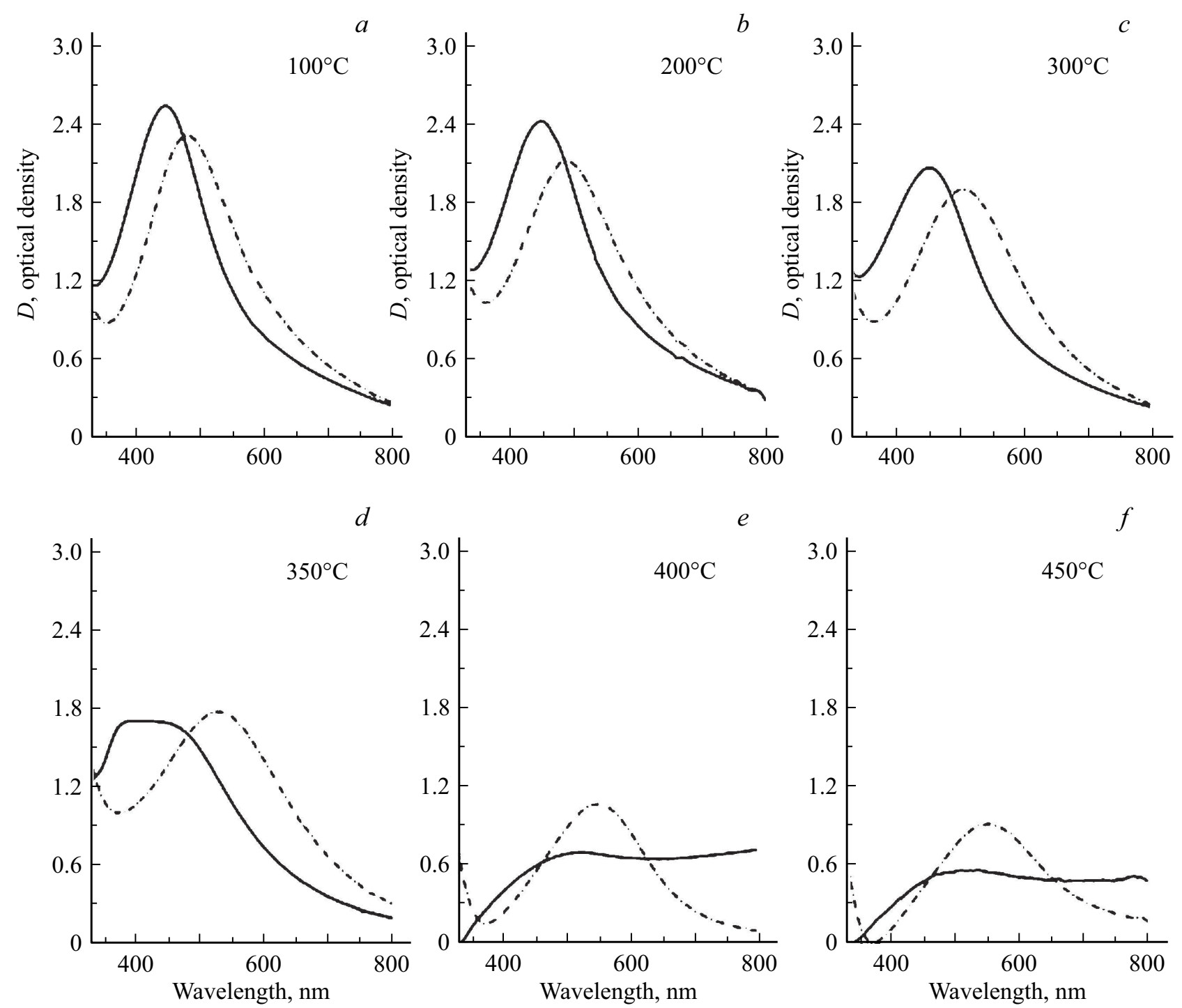

Рис. 1. Типичные спектры оптической плотности нанокомпозитных пленок $a$-C:H $\langle\mathrm{Ag} 14.9$ at.\% (сплошные кривые) и $a$ $\mathrm{C}: \mathrm{H}\langle\mathrm{Ag} 14.9$ at.\%+TiO 2.2 at.\% $\rangle$ (штриховая линия), отожженных при температурах от 100 до $450^{\circ} \mathrm{C}$.

микроскопе (SEM) Quanta 3D 200i с ускоряющим напряжением $30 \mathrm{kV}$. Максимальная концентрация серебра в нанокомпозитных пленках достигала 19 at.\%, а концентрация титана -5 at.\%. Химический состав нанокомпозитных пленок определялся с использованием рентгеновской фотоэлектронной спектроскопии (РФЭС) на установке KRATOS AXIS 165 XPS с монохроматическим рентгеновским источником $\mathrm{Al}_{K \alpha} \mathrm{c}$ энергией $1486.7 \mathrm{eV}$. Спектры РФЭС регистрировались в диапазоне энергий от 0 до $1100 \mathrm{eV}$ с шагом $0.5 \mathrm{eV}$ и погрешностью в пределах $\mp 0.3 \mathrm{eV}$.

Структура нанокомпозитных пленок изучалась $\mathrm{c}$ помощью просвечивающего электронного микроскопа TEM JEM 2100 JEOL с ускоряющим напряжением $100 \mathrm{kV}$ в режиме светлого поля, обработка ТЕМ-изображений проводилась с использованием программы ImageJ.
Отжиг нанокомпозитов $a-\mathrm{C}: \mathrm{H}\langle\mathrm{Ag}\rangle$ и $a-\mathrm{C}: \mathrm{H}\langle\mathrm{Ag}+$ $\left.+\mathrm{TiO}_{2}\right\rangle$ проводился в течение $1 \mathrm{~h}$ в атмосфере аргона в интервале температур от $100^{\circ} \mathrm{C}$ до $450^{\circ} \mathrm{C}$ с шагом $50^{\circ} \mathrm{C}$. Охлаждение нанокомпозитов после отжига проводилось в атмосфере аргона в течение $10 \mathrm{~h}$. Спектры поглощения пленок до и после отжига регистрировались при комнатной температуре на установке Lambda 35 (PerkinElmer) в диапазоне от 300 до $1100 \mathrm{~nm}$ с шагом $1 \mathrm{~nm}$.

\section{Результаты и обсуждение}

На рис. 1 показаны типичные спектры оптической плотности нанокомпозитов $a-\mathrm{C}: \mathrm{H}\langle\mathrm{Ag}\rangle$ и $a-\mathrm{C}: \mathrm{H}\langle\mathrm{Ag}+$ $\left.+\mathrm{TiO}_{2}\right\rangle$ с разными концентрациями диоксида серебра и диоксида титана, отожженных при температурах от 100 до $450^{\circ} \mathrm{C}$. Нанокомпозитные пленки $a-\mathrm{C}: \mathrm{H}\langle\mathrm{Ag}\rangle$ и $a-\mathrm{C}: \mathrm{H}\left\langle\mathrm{Ag}+\mathrm{TiO}_{2}\right\rangle$, отожженные при $100^{\circ} \mathrm{C}$ в аргоне (так 
$a$
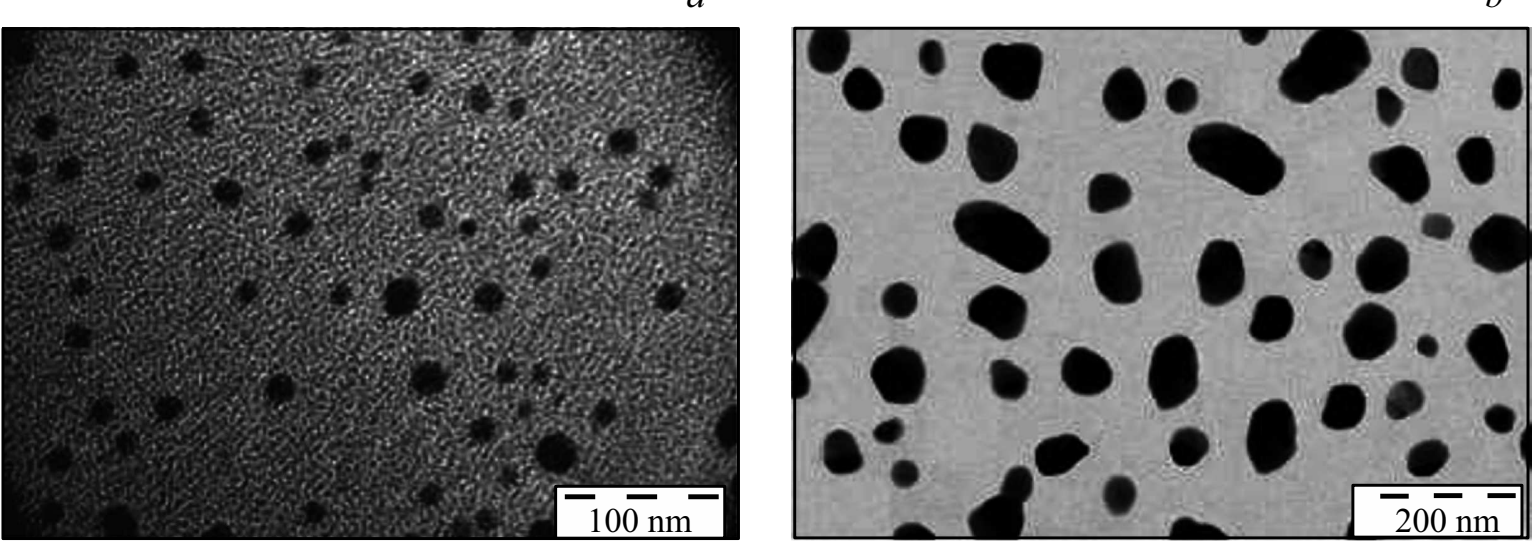

$c$

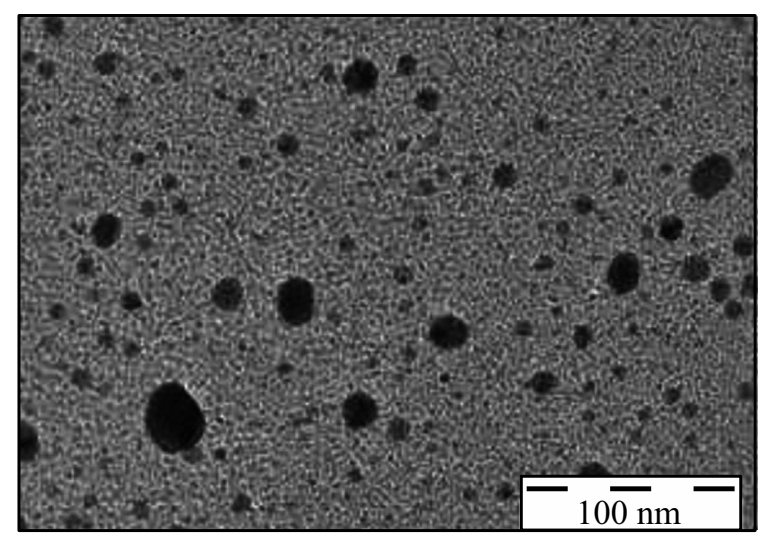

$d$

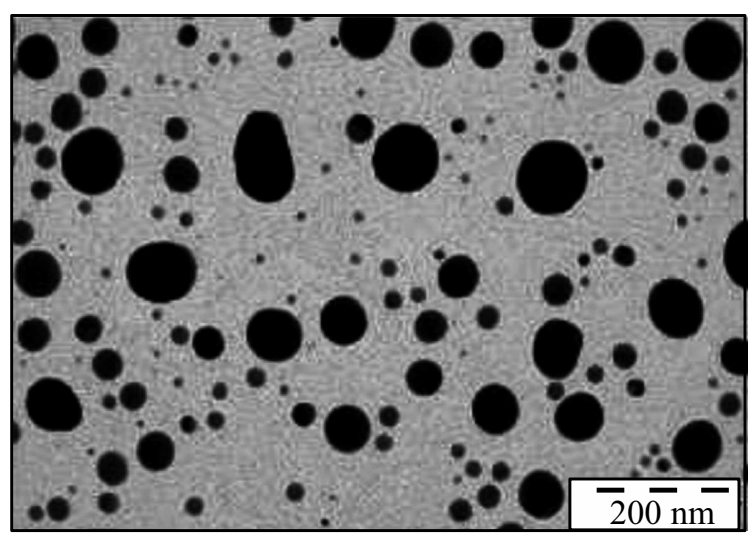

Рис. 2. ТЕМ-изображения нанокомпозитов $a$-C: $\mathrm{H}\left\langle\operatorname{Ag} 14.9\right.$ at.\% $\%(a, b), a-\mathrm{C}: \mathrm{H}\left\langle\mathrm{Ag} 14.9\right.$ at.\% $\% \mathrm{TiO}_{2} 2.2$ at.\% $\%(c, d)$ до $(a, c)$ и после отжига при $350^{\circ} \mathrm{C}(b, d)$.

же, как и свежеприготовленные пленки), имеют ярко выраженный плазмонный резонанс в видимой области спектра. Максимумы ППР этих нанокомпозитных пленок расположены на 443 и $478 \mathrm{~nm}$ соответственно. Интенсивность резонансного поглощения в нанокомпозитных пленках $a$-C: $\mathrm{H}\left\langle\mathrm{Ag}+\mathrm{TiO}_{2}\right\rangle$ примерно на $10 \%$ ниже, чем в $a-\mathrm{C}: \mathrm{H}\langle\mathrm{Ag}\rangle$.

Отжиг при температурах от 100 до $300^{\circ} \mathrm{C}$ приводит к уменьшению интенсивности пиков резонансного поглощения в нанокомпозитных пленках на 19\% и сдвигу их максимумов в длинноволновую область спектра. Последнее, по-видимому, связано с изменением диэлектрической проницаемости матрицы нанокомпозитов $a-\mathrm{C}: \mathrm{H}\langle\mathrm{Ag}\rangle$ и $a-\mathrm{C}: \mathrm{H}\left\langle\mathrm{Ag}+\mathrm{TiO}_{2}\right\rangle$ в результате графитизации. Увеличение температуры отжига от 300 до $450^{\circ} \mathrm{C}$ для нанокомпозитных пленок $a-\mathrm{C}: \mathrm{H}\langle\mathrm{Ag}\rangle$ приводит к существенным изменениям их спектров ППР. А именно отжиг при $350^{\circ} \mathrm{C}$ приводит к заметной трансформации пика ППР в широкое „плато“. Дальнейшее повышение температуры отжига до $400^{\circ} \mathrm{C}$ и выше приводит к исчезновению ППР. Этот эффект, по-видимому, связан с коагуляцией наночастиц серебра малого размера, которыми обусловлен ППР, в более крупные наночастицы.
В нанокомпозитных пленках $a-\mathrm{C}: \mathrm{H}\left\langle\mathrm{Ag}+\mathrm{TiO}_{2}\right\rangle$ в отличие от пленок $a$-C:H $\langle\mathrm{Ag}\rangle$ ППР сохраняется после отжига в интервале температур $350-450^{\circ} \mathrm{C}$. Однако в этом случае наблюдаются уменьшение интенсивности ППР и заметный сдвиг пика в длинноволновую область спектра.

Проведено исследование влияния отжига на структуру нанокомпозитных пленок $a$-C:H $\langle\mathrm{Ag}\rangle \quad$ и $a-\mathrm{C}: \mathrm{H}\left\langle\mathrm{Ag}+\mathrm{TiO}_{2}\right\rangle$. На рис. 2 и 3 приведены ТЕМ-изображения и соответствующие гистограммы диаметров наночастиц до и после отжига при $350^{\circ} \mathrm{C}$. Можно видеть, что отжиг приводит к появлению крупных наночастиц в структуре нанокомпозитов. Очевидно, что эти большие наночастицы являются результатом слияния более мелких наночастиц серебра при отжиге.

Существенно, что наночастицы с диаметром менее $30 \mathrm{~nm}$ в отожженных нанокомпозитах $a$-C:H $\langle\mathrm{Ag}\rangle$ почти отсутствуют (рис. 2,b и 3,b). С другой стороны, в нанокомпозитах $a-\mathrm{C}: \mathrm{H}\left\langle\mathrm{Ag}+\mathrm{TiO}_{2}\right\rangle$ (рис. $2, d$ и $3, d$ ) после отжига наблюдаются наночастицы малого размера, которые являются наночастицами как серебра, так и диоксида титана $[24,25]$. Можно предположить, что после отжига нанокомпозитов $a-\mathrm{C}: \mathrm{H}\left\langle\mathrm{Ag}+\mathrm{TiO}_{2}\right\rangle$ при $350^{\circ} \mathrm{C}$ ППР 

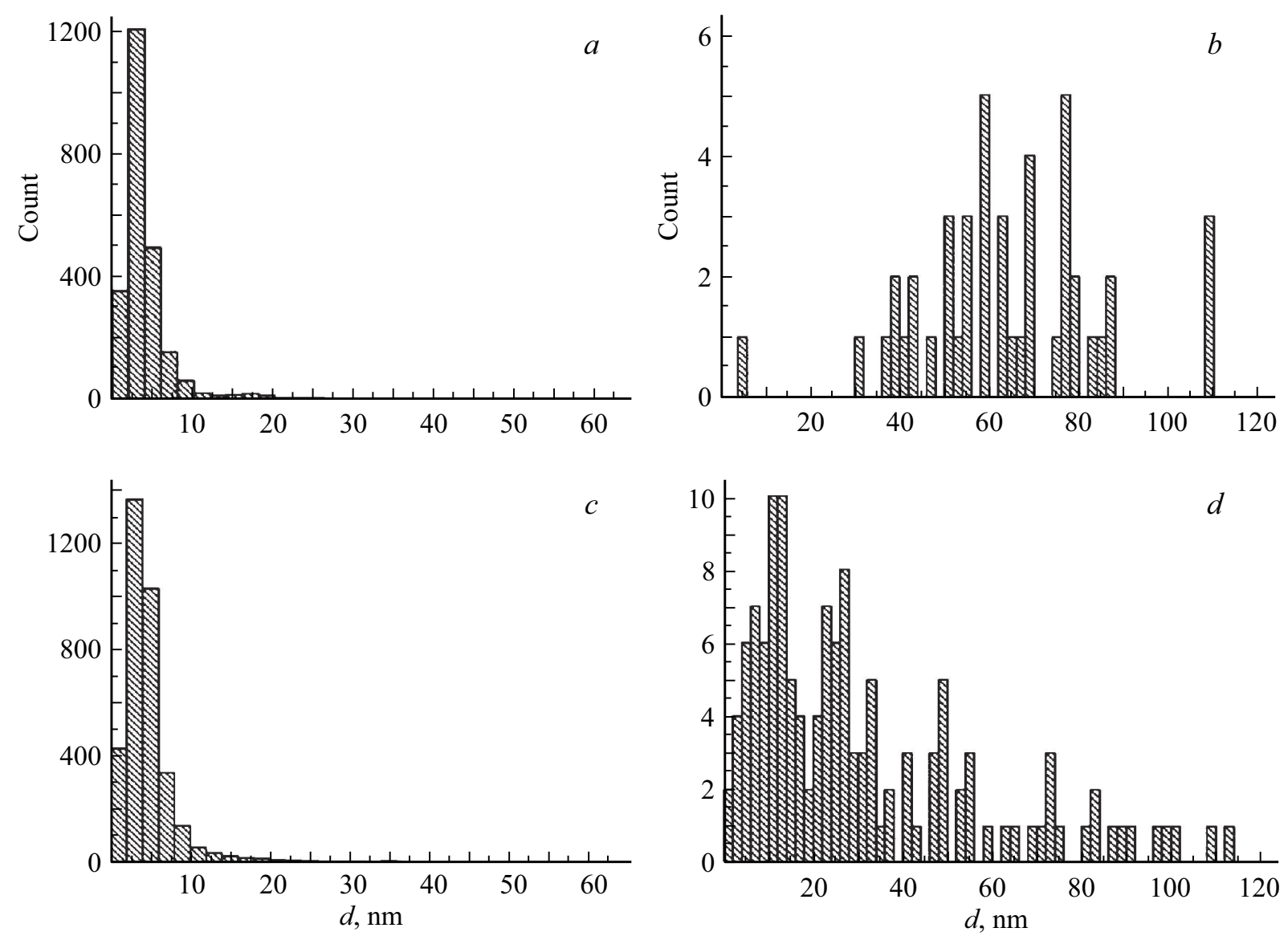

Рис. 3. Распределение диаметров наночастиц для нанокомпозитов $a$-C: $\mathrm{H}\langle\mathrm{Ag} 14.9$ at.\% $\rangle \quad(a, b), \quad a-\mathrm{C}: \mathrm{H}\langle\mathrm{Ag} 14.9$ at.\%+ $+\mathrm{TiO}_{2} 2.2$ at.\% $(c, d)$ до $(a, c)$ и после $(b, d)$ отжига при $350^{\circ} \mathrm{C}$, полученные из ТЕМ-изображений.

происходит из-за присутствия мелких наночастиц Ag. Отметим, что диаметр наночастиц $\mathrm{TiO}_{2}$ при выбранных температурах отжига не изменился.

Для оценки среднего диаметра $d$ наночастиц $\mathrm{Ag}$, на которых происходит ППР в исследуемых нанокомпозитных пленках, используем теорию рассеяния Ми для гетерогенных сред, состоящих из диэлектрической матрицы с включениями изолированных металлических наночастиц [26]. Теория справедлива для наночастиц с размерами менее $\lambda / 20$ (где $\lambda$ - длина волны падающего света). Средний диаметр $d$ наночастицы может быть оценен по полуширине пика резонансного оптического поглощения $\Delta \lambda$ и характеристической длине волны плазмонного резонанса $\lambda_{p}[26]$ :

$$
d=\frac{v_{f} \lambda_{p}^{2}}{\pi c \Delta \lambda}
$$

где $v_{f}$ - скорость электронов на уровне Ферми в металле (для серебра $v_{f}=1.39 \cdot 10^{8} \mathrm{~cm} / \mathrm{s}$ ), $c$ - скорость света. Вычисленный $d$ для наночастиц $\mathrm{Ag}$ в свежеприготовленных нанокомпозитах $a-\mathrm{C}: \mathrm{H}\langle\mathrm{Ag}\rangle$ и $a-\mathrm{C}: \mathrm{H}\left\langle\mathrm{Ag}+\mathrm{TiO}_{2}\right\rangle$ составляет $\sim 2.7 \mathrm{~nm}$, после отжига нанокомпозитов он остается практически таким же (рис. 4).

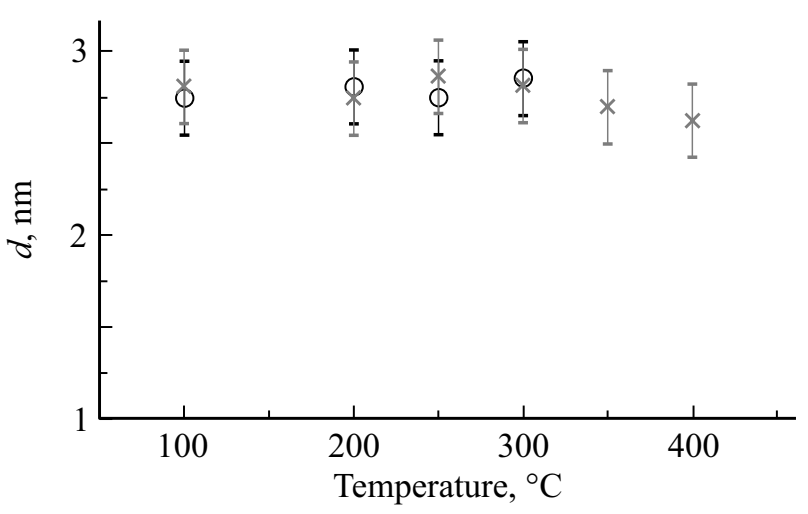

Рис. 4. Средний диаметр наночастиц серебра в нанокомпозитах $a-\mathrm{C}: \mathrm{H}\langle\mathrm{Ag} 14.9$ at.\% $\%$ (кружки), $a-\mathrm{C}: \mathrm{H}\langle\mathrm{Ag} 14.9$ at.\%+ $+\mathrm{TiO}_{2} 2.2$ at.\% $\%$ (крестики) при разных температурах отжига, вычисленный по теории Ми.

Анализ результатов, представленных на рис. 1, показывает, что ППР в нанокомпозитных пленках $a-\mathrm{C}: \mathrm{H}\left\langle\mathrm{Ag}+\mathrm{TiO}_{2}\right\rangle$ является более термически стабильным по сравнению с ППР в пленках $a-\mathrm{C}: \mathrm{H}\langle\mathrm{Ag}\rangle$. Можно предположить, что коагуляция наночастиц серебра ингибируется из-за присутствия диэлектрических наночастиц 
из диоксида титана в нанокомпозитах $a$-C: $\mathrm{H}\left\langle\mathrm{Ag}+\mathrm{TiO}_{2}\right\rangle$. Аналогичный вывод был сделан в [27], где коалесценция наночастиц серебра при высоких температурах значительно замедлилась в присутствии диэлектрических наночастиц $\mathrm{SiO}_{2}$.

Таким образом, ППР в нанокомпозитах $a-\mathrm{C}: \mathrm{H}\langle\mathrm{Ag}+$ $\left.+\mathrm{TiO}_{2}\right\rangle$ в отличие от нанокомпозитов $a-\mathrm{C}: \mathrm{H}\langle\mathrm{Ag}\rangle$ coxpaняется при существенно более высоких температурах.

\section{Заключение}

В нанокомпозитных пленках $a-\mathrm{C}: \mathrm{H}\langle\mathrm{Ag}\rangle$, полученных ионно-плазменным магнетронным распылением, проявляется ярко выраженный ППР в спектрах оптической плотности, который сохраняется до температуры отжига $350^{\circ} \mathrm{C}$. В нанокомпозитах $a-\mathrm{C}: \mathrm{H}\left\langle\mathrm{Ag}+\mathrm{TiO}_{2}\right\rangle$, полученных тем же методом, ППР сохраняется до гораздо более высоких температур отжига, т. е. до $450^{\circ} \mathrm{C}$. Таким образом, ППР в нанокомпозитных пленках $a-\mathrm{C}: \mathrm{H}\left\langle\mathrm{Ag}+\mathrm{TiO}_{2}\right\rangle$ является более термически стабильным.

Термостабильность поверхностного плазмонного резонанса в нанокомпозитах $a-\mathrm{C}: \mathrm{H}\left\langle\mathrm{Ag}+\mathrm{TiO}_{2}\right\rangle$ при температурах выше $350^{\circ} \mathrm{C}$, по-видимому, обусловлена присутствием наночастиц $\mathrm{TiO}_{2}$, которые ингибируют коалесценцию наночастиц серебра.

Авторы выражают благодарность В.Н. Мочалину (Миссурийский университет науки и технологий, Ролла, Миссури, США) за плодотворную дискуссию при обсуждении результатов эксперимента.

Исследования проведены в рамках гранта № AР05132897 Комитета науки МОН РК.

\section{Список литературы}

[1] Yeshchenko O.A., Dmitruk I.M., Alexeenko A.A., Kotko A.V., Verdal J., Pinchuk A.O. // Plasmonics. 2012. V. 7. P. 685.

[2] Sarsembinov Sh., Prikhodko O., Ryaguzov A., Maksimova S.Ya., Daineko Ye.A., Mahmoud F.A. // Phys. Status Solidi C. 2010. V. 7. P. 805.

[3] Muradov A.D., Mukashev K.M., Yar-Mukhamedova G.Sh., Korobova N.E. // Technical Physics. 2017. V. 62. P. 1692.

[4] Pena-Rodríguez O., Pal U. // Nanoscale Res. Lett. 2011. V. 6. P. 279.

[5] Lee K., El-Sayed M.A. // J. Phys. Chem. B. 2006. V. 110. P. 19220.

[6] Hedayati M.K., Faupel F., Elbahri M. // Materials. 2014. V. 7. P. 1221.

[7] Okumu J., Kohl D., Sprafke A., Von Plessen G., Wuttig M. // J. Appl. Phys. 2010. V. 108. P. 063529.

[8] Kumar M., Parashar K.K., Tandi S.K., Kumar T., Agarwal D.C., Pathak A. // J. Spectrosc. 2013. V. 2013. P. 491716.

[9] Ohko Y., Tatsuma T., Fujii T., Naoi K., Niwa C., Kubota Y., Fujishima A. // Nat. Mater. 2003. V. 2. P. 29.

[10] Armelao L., Barreca D., Bottaro G., Gasparotto A., Maccato C., Tondello E., Lebedev O.I., Turner S., VanTendeloo G., Sada C., Stangar U.L. // Chem. Phys. Chem. 2009. V. 10. P. 3249.
[11] Khan S.A., Avasthi D.K., Agarwal D.C., Singh U.B., Kabiraj D. // Nanotechnology. 2011. V. 22. P. 235305.

[12] Garcia-Serrano J., Pal U. // Int. J. Hydrogen Energy. 2003. V. 28. P. 637.

[13] Sernaa R.J., Ballesterosa J.M., Solisa J., Afonsoa C.N., Osborneb D.H., Haglund R.F., Petford-Longc A.K. // Thin Sol. Films. 1998. V. 318. P. 96.

[14] Kumar M., Reddy G.B. // Phys. Stat. Sol. B. 2009.V. 246. P. 2232.

[15] Kumar M., Reddy G.B. // Physica E. 2010. V. 43. P. 470.

[16] Kumar M., Kulriya P.K., Pivin J.C., Avasthi D.K. // J. Appl. Phys. 2011. V. 109. P. 044311.

[17] Manish K., Suchand Sandeep C.S., Kumar G., Mishra Y.K., Philip R., Reddy G.B. // Plasmonics. 2014. V. 9. P. 129.

[18] Meškinis Š., Čiegis A., Vasiliauskas A. // Nanoscale Res. Lett. 2016. V. 11. P. 146.

[19] Kreibig U., Vollmer M. Optical properties of metal clusters. Springer, 1995. $535 \mathrm{p}$.

[20] Stalmashonak A., Seifert G., Abdolvand A. Ultra-Short Pulsed Laser Engineered Metal-Glass Nanocomposites. Springer Briefs in Physics, 2013. 70 p.

[21] Yeshchenko O.A., Bondarchuk I.S., Gurin V.S., Dmitruk I.M., Kotko A.V. // Surf. Sci. 2013. V. 608. P. 275.

[22] Maier S.A. Plasmonics: fundamentals and applications. Springer US, 2007. 224 p.

[23] Kumar M., Kumar T., Avasthia D.K. // Script. Mater. 2015. V. 105. P. 46.

[24] Prikhodko O.Yu., Mikhailova S.L., Mukhametkarimov Ye.S., Maksimova S.Ya, Manabaev N.K., Dauthan K. // Proc. SPIE. 2016. V. 9929. P. 99291G-1.

[25] Приходько О.Ю., Михайлова С.Л., Мухаметкаримов Е.С., Даутхан К., Максимова С. Я. // Опт. спектр. 2017. T. 123. C. 353; [Prikhodko O., Mikhailova S., Mukhametkarimov Ye., Dauthan K., Maksimova S. // Opt. Spectrosc. 2017. V. 123. P. 383.]

[26] Manikandan D., Mohan S., Nair K.G. // Mater. Res. Bull. 2003. V. 38. P. 1545.

[27] Kim Y.H., Kim C.W., Cha H.G., Lee D.K., Jo B.K., Ahn G.W., Hong E.S., Kim J.C., Kang Y.S. // J. Phys. Chem. 2009. V. 113. P. 5105 . 